\title{
Fit für die Zukunft! Phytopharmaka müssen attraktiver werden
}

\author{
Nicole Armbrüster ${ }^{1}$, René Roth-Ehrang ${ }^{2}$ \\ 1 Bundesverband der Pharmazeutischen Industrie e. V., Berlin \\ 2 Finzelberg $\mathrm{GmbH} \&$ Co. KG, Andernach
}

\section{ZUSAMMENFASSUNG}

Phytopharmaka sind fester Bestandteil der Gesundheitsversorgung. Sie spielen insbesondere bei der Selbstmedikation eine große Rolle. Durch fehlende Anreize für Innovationen und Weiterentwicklungen sowie stetig wachsende regulatorische Anforderungen und die Konkurrenz durch andere Produktgruppen wird ihr Erhalt mehr und mehr gefährdet. Im Folgenden werden Lösungen vorgestellt, mit denen Phytopharmaka für Hersteller wieder attraktiver werden was auch in Zukunft die Therapievielfalt sicherstellen würde.

\section{Einführung}

Phytopharmaka besitzen in der Selbstmedikation einen hohen Stellenwert und sind fester Bestandteil der Gesundheitsversorgung. Insbesondere bei Erkältungskrankheiten, mentalem Stress, Stimmungsschwankungen, Harnwegserkrankungen oder Magen-Darm-Beschwerden werden sie häufig erfolgreich angewendet [1].

Gerade weil pflanzliche Arzneimittel oft besser verträglich sind und das Risiko für Nebenwirkungen gering ist, sehen viele Patientinnen und Patienten in ihnen eine Alternative zu Arzneimitteln mit chemisch-synthetischen Wirkstoffen [2].

Solchen Befunden zum Trotz sind Phytopharmaka in Gefahr. Ihre Produktion ist stetig wachsenden regulatorischen Anforderungen unterworfen. Wie sieht die Zukunft pflanzlicher Arzneimittel aus der Perspektive der Hersteller aus? Darum geht es in diesem Beitrag.

\section{Der Markt für Phytopharmaka}

Neben Frankreich und Polen ist Deutschland der wichtigste Markt für Phytopharmaka in Europa. Mit Phytopharmaka wurden in Deutschland im Jahr 2020 circa 1,4 Milliarden Euro umgesetzt, davon wurden $92 \%$ für den Kauf pflanzlicher Arzneimittel in der Selbstmedikation aufgebracht.

Der deutsche Markt für Phytopharmaka blieb in den letzten Jahren konstant. Im Jahr 2020 nahm die Zahl der in der Apotheke ausgegebenen Packungseinheiten im Vergleich zum Vorjahr, sicher auch coronabedingt, um circa $12 \%$ ab ( $\triangleright$ Abb. 1). Die weitere Entwicklung bleibt abzuwarten.

\section{Anzahl der Phytopharmaka sinkt}

Trotz der großen Beliebtheit pflanzlicher Arzneimittel in der Bevölkerung kann bei näherer Betrachtung festgestellt werden, dass es keinen Grund zur Euphorie gibt. Wie erwähnt war der Markt in den Jahren vor 2020 zwar stabil, aber wenig dynamisch. Innovationen auf Basis neuer pflanzlicher Wirkstoffe sind selten, wie der Blick auf die BfArM-Statistik zu verkehrsfähigen zugelassenen und registrierten Phytopharmaka zeigt. Belief sich deren Anzahl Anfang 2016 auf insgesamt 1719, so war sie 5 Jahre später bereits auf 1356 gesunken, was einer Abnahme von circa $21 \%$ entspricht.

Auch das vermeintlich vereinfachte Verfahren der traditionellen Registrierung (TUR) verlor an Attraktivität. Dies zeigen die Zahlen des Komitees für pflanzliche Arzneimittel (HMPC) der Europäischen Arzneimittelagentur ( Abb. 2). Nach Inkrafttreten der Richtlinie 2004/24 / EG zur Schaffung eines Gemeinschaftskodexes für Humanarzneimittel hinsichtlich traditioneller pflanzlicher Arzneimittel im Jahr 2004, nahm die Anzahl der jährlichen Registrierungen zunächst stetig zu, bis 2011 ein Peak erreicht war, danach sank sie deutlich. Ein Grund dafür könnte das Votum für die erste Liste genehmigter gesundheitsbezogener Angaben (Health Claims) und die Aussicht der Nutzung der sogenannten on hold-Claims für gesundheitsbezogene Aussagen für pflanzliche Stoffe in Lebensmitteln sein. Zudem endete 2011 die Übergangsfrist für die Registrierung traditioneller pflanzlicher Arzneimittel (THMP), die bereits vor der Einführung der Richtlinie auf dem Markt erhältlich waren. Der ebenso auffällige Rückgang ab 2014 mag mit der Bestätigung der on holdClaims in Zusammenhang stehen. Leider führt das HMPC die Statistik seit 2017 nicht weiter. 


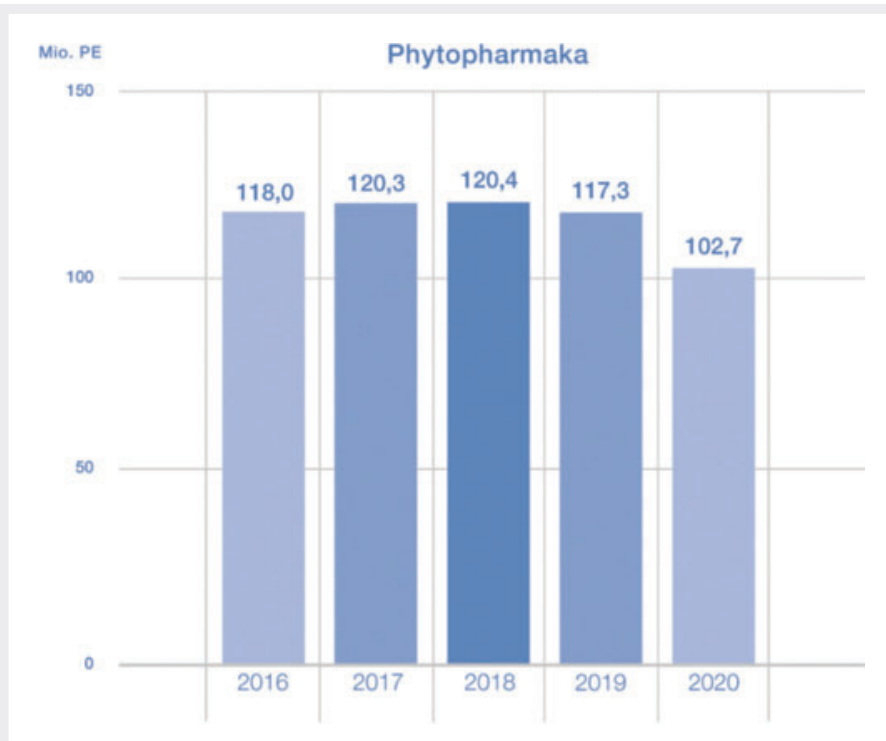

-Abb. 1 Absatzentwicklung im Apothekenmarkt 2016 bis 2020 (in Millionen Packungseinheiten [PE]). Quelle: Darstellung des BPI, basierend auf Daten von IQVIA ${ }^{\mathrm{TM}}$, IMS ${ }^{\circledR}$ OTC / GMS und IMS ${ }^{\circledR}$ Pharmascope National 2021

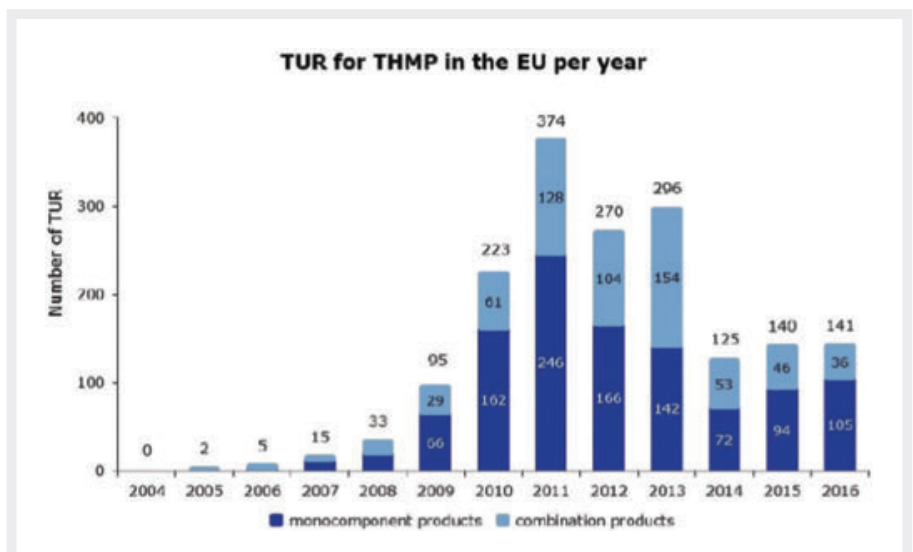

-Abb.2 Anzahl Registrierungen traditioneller pflanzlicher Arzneimittel (TUR) in der EU, gruppiert nach Jahr der Registrierung von Mono- und Kombinationspräparaten (2004 bis Dezember 2016) [1].

\section{Was sind die Gründe?}

\section{Stolpersteine für Innovationen}

Aus Erhebungen des Bundesverbandes der Pharmazeutischen Industrie geht hervor, dass nur noch wenige Hersteller in die Forschung und Entwicklung neuer Phytopharmaka oder in die Erweiterung von Indikationen oder Anwendungsgebieten bekannter Stoffe investieren [3]. In den letzten Jahren gab es daher nur wenige Innovationen bei bewährten pflanzlichen Arzneimitteln und kaum neue pflanzliche Arzneimittel mit neuen Wirkstoffen. Als Gründe hierfür sind der lange Zeitraum für die
Entwicklung neuer Arzneimittel, die erheblichen regulatorischen Hürden und die damit verbundenen hohen Kosten anzuführen.

Im Vergleich zu chemisch definierten Wirkstoffen gelten bei Phytopharmaka neue Extrakte (auch) aus bekannten Pflanzen als neue Wirkstoffe. Damit unterliegen sie automatisch der Verschreibungspflicht und der Nutzenbewertungspflicht beim Gemeinsamen Bundesausschuss (G-BA). Dies ist nach Meinung vieler eine nutzlose Regelung, die sowohl Kapazitäten bindet und die Zeit bis zur Markteinführung erheblich verlängert als auch die Ausgaben der Hersteller nach oben treibt. Sie gilt, obwohl die Arzneimittel nach kurzer Dauer regelmäßig wieder aus der Verschreibungspflicht entlassen werden und keine Belastung für die gesetzliche Krankenversicherung (GKV) bedeuten.

Auch Weiterentwicklungen bereits bekannter Wirkstoffe sind schwierig und wenig attraktiv, weil z. B. bei einer Indikationserweiterung, für die in aufwendigen Studien klinische Daten erhoben werden müssen, kein ausreichender Unterlagenschutz gewährt wird. Aktuell gibt es keine Anreize für die Generierung neuer wissenschaftlicher Daten.

\section{Regulatorische Hürden}

Ebenfalls keinen Raum für Innovationen lässt der immer höhere Aufwand für die Aufrechterhaltung bereits vorhandener Registrierungen und Zulassungen. So verlagerte sich der Anteil der Ressourcenbindung in den Zulassungsabteilungen der Unternehmen stetig in Richtung der Pflege bestehender Zulassungen und Registrierungen. Insbesondere ist die aktuelle Variation Guideline eine wachsende Herausforderung. Die Anforderungen an das Änderungsmanagement sind für Phytopharmaka teilweise sogar noch höher als für chemisch-synthetische Arzneimittel und für die Besonderheiten der Phytopharmaka in vielen Aspekten nicht sachgerecht. Zum Beispiel wird die Änderung der geografischen Quelle der Drogenherkunft als Variation Type II eingestuft, obwohl sich an den Spezifikationen keine Änderungen ergeben und die gleiche Qualität stets zu gewährleisten ist.

Vor dem Hintergrund immer extremerer Klimaphänomene ist davon auszugehen, dass Ernteausfälle oder Verknappungen in einzelnen Regionen noch öfter vorkommen. Mit dem starren System und der Einstufung als zustimmungspflichtige Änderung sind häufigere Lieferengpässe, wie es sie in den vergangenen Jahren vereinzelt gab, vorprogrammiert.

Die politisch und klimatisch bedingten Ausfallrisiken bedürfen einer größeren Flexibilität und schnellerer Verfahren. Aber auch technische Änderungen und Weiterentwicklungen oder die Entwicklung alternativer Darreichungsformen erfordern einen immensen 
Aufwand für das Änderungsmanagement, dem sich ein Unternehmen risikoabwägend stellen muss.

Eine Ungleichbehandlung zu chemisch-synthetischen Arzneimitteln erfahren Phytopharmaka durch die Erstellung der Monografien durch das HMPC. Diese sollen der Harmonisierung in Europa dienen, sind aber leider oft Stolpersteine. Zwar sind solche Monografien und Bewertungsmaßstäbe mit allen Gesundheitsbehörden abgestimmt, jedoch besitzen sie keine zwingende Verbindlichkeit für die zuständigen Behörden. Die mit der Richtlinie 2004 / 24 / EG angestrebte Harmonisierung ist gescheitert, was eine Evaluierung der Europäischen Kommission bestätigt [4].

Hinzu kommt, dass in den Monografien des HMPC zunehmend Aussagen zur Unbedenklichkeit und Anwendung, insbesondere bei Kindern und Jugendlichen, eingeschränkt oder Indikationen sogar im Ganzen gestrichen werden. Auch ist die Akzeptanz klinischer Daten für eine „anerkannte medizinische Verwendung“ (well-established use) zwischen den Mitgliedstaaten nicht immer einheitlich.

\section{Konkurrenz anderer Produktkategorien}

Gleichzeitig stehen Phytopharmaka in Konkurrenz mit anderen pflanzlichen Gesundheitsprodukten, in denen Pflanzen und pflanzliche Zubereitungen eingesetzt werden. Zu ihnen zählen Kosmetika, Medizinprodukte und insbesondere Lebens- bzw. Nahrungsergänzungsmittel (NEM). Für diese Produktkategorien gibt es deutlich weniger regulatorische Anforderungen als im Arzneimittelbereich, sodass neue innovative NEM-Produkte innerhalb kurzer Zeit zur Marktreife gelangen können. Firmen empfinden diesen Weg zur Produkteinführung inzwischen als attraktiv, da er viel weniger Risiken birgt sowie planbarer und kostengünstiger ist. Unterstützt wird dies durch die Aussetzung der sogenannten Health-ClaimsVerordnung (VO (EG) 1924/2006), die die Bewertung von Health Claims zu pflanzlichen Stoffen in Lebensmitteln zum Ziel hat. Aufgrund von Übergangsregelungen können Produkte mit noch nicht bewerteten Health Claims auf dem Markt verbleiben. Die Abgrenzung zwischen den Produktkategorien ist damit schwierig und deren Merkmale hinsichtlich ihrer Wirksamkeit, Qualität und Sicherheit für Verbraucher und Verbraucherinnen schwer erkennbar.

Immer mehr Hersteller pflanzlicher Arzneimittel interessieren sich zunehmend für einen alternativen Marktzugang für ihre Produkte. Schließlich gibt es kaum noch Abgrenzungsmerkmale zu den NEM, die insbesondere bei THMP einen Vorteil für die Vermarktung bedeuten. Bereits jetzt ist der Trend erkennbar, dass viele pflanzliche Arzneimittel aus wirtschaftlichen Gründen vom Markt genommen werden oder sich in weniger stark regulierten Produktkategorien wie NEM oder Kosmetika wiederfinden.

\section{Wissen über Phytopharmaka geht verloren}

In medizinischen Fachkreisen ist der Kenntnisstand zur Phytotherapie beklagenswert gering. In den Aus-, Fortund Weiterbildungen für ärztliches Personal hat die Phytotherapie einen zu niedrigen Stellenwert. Selbst im Medizinstudium ist sie schwach verankert: Nur an 13 medizinischen Fakultäten ist Phytotherapie Teil der Lehre, 12 hielten kein Lehrangebot bereit, bei den übrigen Fakultäten war das Angebot nicht online verfügbar [5]. Auch wenn ein Lehrangebot vorhanden war, erfolgte meist nur die Vermittlung von Basiskenntnissen. Daher ist davon auszugehen, dass die überwiegende Zahl der heute Medizinstudierenden am Ende ihres Studiums keine oder allenfalls Basiskenntnisse zur Phytotherapie haben werden. Das Angebot reicht nicht aus, damit sich künftige Generationen von Ärztinnen und Ärzten mit der Phytotherapie differenziert befassen können. Dies wird der Bedeutung der Phytotherapie nicht gerecht!

Zu einem ähnlichen Resultat kommt eine Studie, bei der neben dem Lehrangebot medizinischer Fakultäten die Phytotherapie im Pharmaziestudium untersucht wurde, wo die Situation besser zu sein scheint: Die Vermittlung fachlichen Wissens über Phytopharmaka wird mit „recht umfassend“ beurteilt [6]. Allerdings weist der Verfasser darauf hin, dass es in der Pharmazeutischen Biologie teilweise zu einer Konkurrenz zwischen der Pharmakognosie und molekularbiologischen Themen kommt. Letztere gewinnen an Bedeutung, da sie insbesondere in der Forschung einen hohen Stellenwert haben.

\section{Lösungswege}

\section{Es ginge auch anders}

Während andere Regionen ihre traditionellen Therapien und Arzneimittel fördern und weiterentwickeln, scheint der Westen traditionelles Wissen und Erfahrungen mit der Phytotherapie und pflanzlichen Arzneimitteln zunehmend gering zu schätzen. Dass es anders gehen kann, zeigt China, wo die Traditionelle Chinesische Medizin (TCM) modernisiert und für die westliche Welt attraktiv gemacht werden soll [7]. Schon 2009 beschloss man dort, die TCM nach modernen Standards weiterzuentwickeln [8]. Dies betrifft z. B. pflanzliche Arzneimittel, die in der Vergangenheit von Patientinnen und Patienten als Teezubereitungen in einem mehrstufigen Verfahren zubereitet wurden. Die recht umständliche und wegen der individuellen Zubereitung zu Hause wenig standardisierte Praxis weicht immer öfter Teegranulaten, die den modernen Anforderungen an die Herstellung pflanzlicher Arzneimittel in nichts nachstehen. 
Europa hingegen scheint die Modernisierung traditioneller Arzneimittel, aber auch die Forschung an neuen Phytopharmaka und deren Entwicklung schwerzufallen: In einem überregulierten System erfahren Forschung, Entwicklung und Modernisierung wenig Unterstützung.

\section{Wie können Lösungen aussehen?}

Zunächst sind regulatorische Vereinfachungen wichtig. Hierzu gehört die Schaffung von Anreizen für die Generierung neuer wissenschaftlicher Daten, z. B. in Form eines verbesserten Unterlagenschutzes. Bei der automatischen Verschreibungspflicht, aber auch für die Nutzenbewertungspflicht bei neuen pflanzlichen Extrakten bedarf es einer Ausnahme bei der Erstattungs- und Dossierpflicht (Opt-out-Regelung). Hierauf muss das Sozialgesetzbuch V (SGB V) angepasst werden.

Die Anerkennung neuen Wissens auch über Indikationserweiterungen oder Ausweitungen der Altersgruppen muss möglich sein, ohne dass Hersteller von well-established use-Präparaten oder THMP regulatorisch zu einer Neuzulassung gezwungen werden-gleiche Dosierung und eine vergleichbare Darreichungsform vorausgesetzt.

Ferner muss die von den Zulassungsbehörden geforderte Detailtiefe bei der Qualitätsdokumentation im Rahmen von Zulassungs- und Registrierungsverfahren für diese Produktgruppe angemessen bleiben. Mit Änderungsanzeigen, die in den letzten Jahren deutlich zugenommen haben, muss der Gesetzgeber pragmatisch und mit Augenmaß umgehen, damit sie den Besonderheiten pflanzlicher Arzneimittel gerecht werden können. Nolte et al. befassten sich umfassend mit einer Verschlankung der Zulassungsdokumentation, insbesondere für das Qualitätsmodul. Sie erarbeiteten eine Reihe von Vorschlägen [9].

Die Attraktivität pflanzlicher Arzneimittel würde durch die automatisch gegenseitige Anerkennung von Zulassungen und Registrierungen der EU-Mitgliedstaaten deutlich gestärkt. Zwar nehmen pharmazeutische

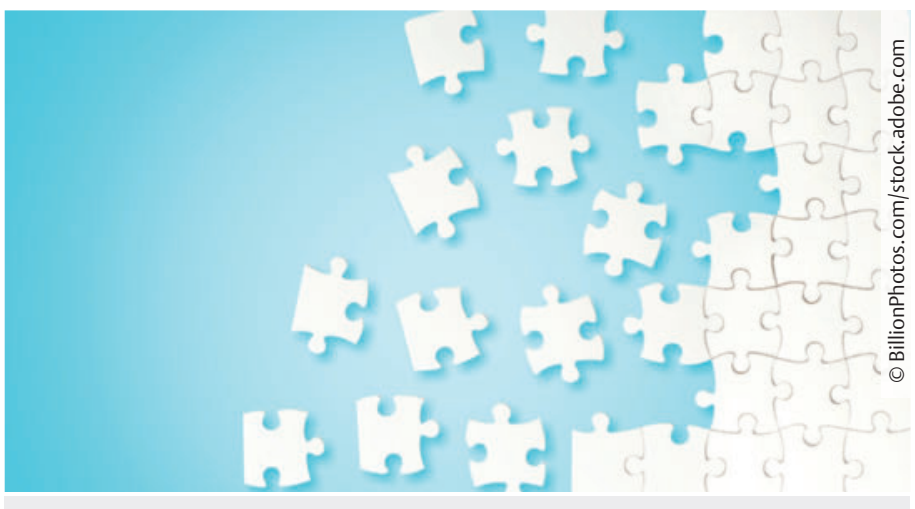

-Abb.3 Eine leichtere gegenseitige Anerkennung von Zulassungen und Registrierungen in der EU wäre ein Gewinn für die Anwendung pflanzlicher Arzneimittel. @ BillionPhotos.com / stock.adobe.com
Unternehmer MRP- und DCP-Verfahren immer häufiger in Anspruch, jedoch sind die Verfahren so aufwendig und zeit- und kostenintensiv, dass bei Zulassungen und Registrierungen noch immer nationale Verfahren die größte Rolle spielen. Eine solche gegenseitige Anerkennung wäre ein wichtiger Beitrag zur Entlastung der Unternehmen und bedeutete eine deutliche Steigerung der Attraktivität der Herstellung von Phytopharmaka ( Abb. 3).

Der wachsenden Konkurrenz durch andere Produktkategorien sollte durch eine klare Abgrenzung entgegengewirkt werden. Eine Möglichkeit wäre es z.B., stärkere Indikationsformulierungen in HMPC-Monografien im Vergleich zu Health Claims für Nahrungsergänzungsmittel zu verankern; sie ermöglichten zudem Verbraucherinnen und Verbrauchern die bessere Unterscheidbarkeit von anderen Produktgruppen. Auch die vollständige Umsetzung der Health-Claims-Verordnung und die Weiterbewertung der Health Claims für pflanzliche Stoffe in Lebensmitteln trügen dazu bei. Oberstes Ziel ist die Stärkung der Phytopharmaka und deren klare Abgrenzung von Lebensmitteln.

Die in Deutschland etablierte Phytotherapie ist eine wichtige Säule unseres Gesundheitssystems. Wissen, das in den letzten Jahrzehnten systematisch gewachsen ist, aber auch gewonnene Erkenntnisse müssen in der medizinischen und pharmazeutischen Ausbildung stärker verankert werden. Deshalb ist die Einrichtung staatlich finanzierter Lehrstühle und akademischer Forschungsinstitute an deutschen Universitäten und die stärkere Unterstützung universitärer Forschungsaktivitäten auf dem Gebiet der Phytopharmaka und -therapie notwendig. In Zukunft müssen bei der Festlegung der Inhalte in Forschung und Lehre der Pharmazeutischen Biologie klassische phytotherapeutische Themen angemessen berücksichtigt werden.

\section{Fazit}

Wir brauchen Phytopharmaka - es sind hochwertige, sichere und wirksame Arzneimittel. Sie sind ein bedeutender Teil der Pharmakotherapie, insbesondere im Bereich der Selbstmedikation, und für Patienten und Patientinnen eine wichtige Säule der Therapievielfalt. Daher müssen für zukünftige Herausforderungen Lösungen gefunden werden - ganz pragmatisch und vor allem jetzt. Nur so wird die Herstellung pflanzlicher Arzneimittel wieder attraktiver. Ihr Fortbestehen und auch ihre Weiterentwicklungen wären somit in unserem Gesundheitssystem gesichert.

\section{ABSTRACT}

Fit for the future! How to get herbal medicines more attractive

Herbal medicines are an integral part of health care and play a particularly important role in self-medication. 
However, due to lack of incentives for innovations and further developments as well as steadily increasing regulatory requirements, their future is increasingly jeopardized. Here, possible solutions are presented how the attractiveness to manufacture herbal medicinal products can be increased and thus the variety of therapies for patients can continue to be secured.

Interessenkonflikt

NA ist Mitarbeiterin des Bundesverbandes der Pharmazeutischen Industrie, der Interessenvertretung der deutschen Pharmaindustrie in Deutschland und Europa. RRE ist Mitarbeiter des Unternehmens Finzelberg GmbH \& Co. KG, das pflanzliche Wirkstoffe sowohl für pflanzliche Arzneimittel als auch für Nahrungsergänzungsmittel herstellt.

\section{Korrespondenzadresse}

\section{Dr. Nicole Armbrüster}

Geschäftsfeldleiterin Biologische / Pflanzliche Arzneimittel, Bundesverband der Pharmazeutischen Industrie e. V. Friedrichstraße 148

10117 Berlin, Deutschland

E-Mail: narmbruester@bpi.de

\section{Literatur}

[1] HMPC. Uptake of the traditional use registration scheme and implementation of the provisions of Directive 2004/24/EC in EU Member States - Status: 31 December 2016, EMA/ HMPC / 322570 / 2011 Rev. 7

[2] Wegener T. Patterns and trends in the use of herbal products, herbal medicine and herbal medicinal products. Int J Complement Altern Med 2017; 9(6): 00317. doi:10.15406/ ijcam.2017.09.00317
[3] Bundesverband der Pharmazeutischen Industrie. Präsentation des BPI im Rahmen der Veranstaltung BfArM im Dialog: Besondere Therapierichtungen - Aktuelle Themen am 26. April 2016. https://www.bfarm.de/SharedDocs/Down loads/DE/Service/Termine-und-Veranstaltungen/dialogver anstaltungen/dialog_2016/160426/06_Folien_Armbruester. pdf?_blob=publicationFile \&v=3

[4] European Commission. Commission Staff Working Document - Evaluation of the Regulation (EC) No 1924/ 2006 on nutrition and health claims made on foods with regard to nutrient profiles and health claims made on plants and their preparations and of the general regulatory framework for their use in foods. 2020. https://ec.europa.eu/food/sites/food/files/safet y/docs/labelling_nutrition-claims_swd_2020-95_part-1.pdf

[5] Kraft K. Lehre der Phytotherapie im Rahmen des Medizinstudiums in Deutschland. Z Phytother 2012; 33: 57-63. doi:10.1055/s-0031-1286047

[6] Erfurth P. Phytotherapie und Naturheilverfahren in der Ausbildung von Medizinern und Pharmazeuten. Z Phytother 2018; 39: 105-111. doi:10.1055/a-0590-9783

[7] The State Council Information Office of the People's Republic of China. Traditional Chinese Medicine in China. White paper. First edition. 2016. http://www.scio.gov.cn/zfbps/32832/Do cument/1534710/1534710.htm

[8] Wang J, Guo Y, Li GL. Current status of standardization of Traditional Chinese Medicine in China. Evid Based Complement Alternat Med 2016; 2016: 9123103. doi:10.1155/2016/ 9123103

[9] Nolte KU, Höhne C, Wagner B et al. Lean Application für pflanzliche Arzneimittel. Pharmind 2018; 80 (2): 176-181

Bibliografie

Zeitschrift für Phytotherapie 2021; 42: 95-99

DOI 10.1055/a-1336-6262

ISSN 0722-348X

(C) 2021. Thieme. All rights reserved.

Georg Thieme Verlag KG, Rüdigerstraße 14,

70469 Stuttgart, Germany 